\title{
Clinical Evaluation of Ramucirumab for the Treatment of Hepatocellular Carcinoma (HCC): Place in Therapy
}

\author{
Khalil Choucair' \\ Syed Kamran' \\ Anwaar Saeed (D) ${ }^{2}$ \\ 'Department of Medicine, Kansas \\ University School of Medicine, Wichita, \\ KS, USA; ${ }^{2}$ Department of Medicine, \\ Division of Medical Oncology, Kansas \\ University Cancer Center, Kansas City, \\ KS, USA
}

\begin{abstract}
Hepatocellular carcinoma remains one of the leading causes of death from cancer worldwide as most cases are diagnosed at an advanced disease stage. Ramucirumab, a human anti-VEGFR-2 monoclonal antibody, is approved as a monotherapy for the treatment of patients with hepatocellular carcinoma and $\alpha$-fetoprotein levels $\geq 400 \mathrm{ng} / \mathrm{mL}$ previously treated with sorafenib. As most patients present with an advanced disease, patients with $\alpha-$ fetoprotein levels $\geq 400 \mathrm{ng} / \mathrm{mL}$ have an aggressive disease and a poor prognosis, making ramucirumab an important treatment option for this subgroup of patients. This article provides a comprehensive review of the clinical efficacy of ramucirumab as highlighted in the two major trials that lead to its approval. We also briefly review the agent pharmacologic properties, as well as its safety and toxicity profile, before discussing certain limitations and challenges associated with ramucirumab use. Finally, we review completed and ongoing clinical trials and focus on those involving ramucirumab-based combinations, namely with immune therapy.
\end{abstract}

Keywords: hepatocellular carcinoma, ramucirumab, targeted therapy

\section{Introduction}

Hepatocellular carcinoma (HCC) is one of the leading causes of cancer deaths worldwide. ${ }^{1}$ Commonly asymptomatic in the early course of the disease, HCC is most diagnosed at an advanced stage, with a relatively poor prognosis and a fiveyear survival rate of $18 \% .^{2}$ Most common risk factors for HCC include nonalcoholic fatty liver disease, alcohol abuse, hepatitis $\mathrm{B}$ or $\mathrm{C}$ virus infection, or any liver disease that causes cirrhosis. ${ }^{2}$ Traditionally, high-risk regions included Japan and parts of China. However, recently the incidence of HCC has increased significantly in European regions and North America, and disproportionately in men ages 55-64 years. ${ }^{3}$ The serum marker alpha-fetoprotein (AFP) is one of the most commonly used serum markers for diagnosis of $\mathrm{HCC}$, and for monitoring treatment response; while elevated AFP levels are not specific to HCC, serum AFP levels $>400 \mathrm{ng} / \mathrm{mL}$ in increased-risk patients are almost diagnostic for HCC. ${ }^{4}$ In terms of prognostic markers, highly elevated AFP levels, vascular invasion, cirrhosis, and multinodular tumors are all predictive of poor outcomes. ${ }^{5}$

Many staging classification systems exist for HCC. A common system is the Barcelona Staging Classification (BCLC) which uses five stages based on the spread of the tumor, the vascularization, and the extent of the primary lesion. ${ }^{6}$
Correspondence: Anwaar Saeed Department of Medicine, Division of Medical Oncology, Kansas University Cancer Center, 2650 Shawnee Mission

Pkwy, Kansas City, KS, 66205, USA

$\mathrm{Tel}+\mid$ 9|3-588-3808

Email asaeed@kumc.edu 
Compared to the classical the Tumor/Node/Metastasis (TNM) system, it provides a more reliable prognostic tool for first-line treatment recommendation, with estimates ranging from three months to greater than five years. ${ }^{7,8}$

Treatment options for HCC are dictated by disease stage. The preferred treatments for HCC are surgical removal and transplantation which can be curative but are commonly not possible due to advanced disease at presentation. When surgical resection is not an option, treatment options are limited to either systemic therapy or liver-directed approaches including microwave or radiofrequency ablation, or embolization. ${ }^{9}$ The choice of correct therapy can be decided using the Child-Turcotte-Pugh Classification, which classifies the severity of liver disease using the levels of ascites, bilirubin, albumin, prothrombin time, and encephalopathy. The most current guidelines favor the atezolizumab plus bevacizumab combination over sorafenib, a multi-tyrosine kinase inhibitor (TKI) that had so far been the standard first-line systemic therapy for patients with advanced HCC. ${ }^{10,11}$ While regorafenib was considered the next-line therapy for patients with disease progression on sorafenib, the most recent National Comprehensive Cancer Network (NCCN) clinical guidelines have also supported the use of ramucirumab in the second-line setting for advanced HCC. ${ }^{12}$

Ramucirumab is a recombinant anti-VEGFR-2 monoclonal antibody that has been approved for treatment in patients with HCC who have failed sorafenib treatment and have an AFP level of $\geq 400 \mathrm{ng} / \mathrm{mL}^{13}$ This article briefly summarizes the pharmacological properties of ramucirumab, provides a detailed review of its therapeutic efficacy and tolerability, and further explores ongoing ramucirumab-based trials and potential future treatment strategies.

\section{Methods}

A systematic review was conducted according to the PRISMA guidelines with the last search update performed on September 30, 2021. The search was conducted in PubMed as well as major conference proceedings (American Society of Clinical Oncology; European Society of Medical Oncology) using the following query terms: (hepatocellular cancer OR liver cancer OR HCC OR primary liver carcinoma) AND (ramucirumab OR cyramza) OR (angiogenesis inhibitors OR anti-VEGF OR anti-VEGFR). In addition, the clinical trials registry (clinicaltrials.gov) was searched to identify ongoing trials that have with so far unpublished reports. Studies were included if they ramucirumab as a monotherapy or in combination with any other agent in a clinical trial setting in patients with advanced HCC. Studies were excluded if they evaluated patients with localized HCC, if a study was a protocol-only publication without data or if it reported overlapping data. In the latter case, the study with the most recent and/or most comprehensive data was included. The initial search identified a total of 6406 studies. After review by title, abstract and full text review, 6 studies were included in the final review (Table 1). Furthermore, one additional ongoing and unpublished study was identified via clinicaltrials.gov.

\section{Ramucirumab: Pharmacology}

Ramucirumab is an IgG1 monoclonal antibody which binds to VEGFR-2 with affinity and acts as a competitive inhibitor of the receptor. This process inhibits downstream signaling pathways that are important for angiogenesis. ${ }^{14}$ Ramucirumab also down-regulates the expression of VEGFR-2. While ramucirumab exerts its anti-angiogenic effects by uniquely and selectively targeting VEGFR-2, other TKIs with anti-angiogenic effects display inhibitory activity by targeting VEGFR-1, 2 and 3, as well as a wider range of receptors with kinase function (platelet-derived growth factor, RAF, follicular growth factor receptor, RET, KIT, MET and AXL).${ }^{15}$ During initial studies, it showed anti-angiogenic and anticancer properties along with a tolerable profile in advanced HCC. ${ }^{16}$ In treatment studies for patients with solid tumors, ramucirumab's target trough level was set at $>20 \mathrm{ug} / \mathrm{mL} .{ }^{17}$ Body weight was found to be an important variable for ramucirumab pharmacokinetics. Initial doses ranged from $6-12 \mathrm{mg} / \mathrm{kg}$ every $2-3$ weeks. Saturation of ramucirumab clearance was seen at $>8 \mathrm{mg} / \mathrm{kg}$, which would indicate that total VEGFR-2 blockage occurred at this dosage. ${ }^{17}$ With this data, Phase 2 trials dosed ramucirumab in patients with $\mathrm{HCC}$ with $8 \mathrm{mg} / \mathrm{kg}$ every two weeks. Steady state was obtained near 12 weeks of therapy. ${ }^{14}$ In the US, dosage is $8 \mathrm{mg} / \mathrm{kg}$ every two weeks via an intravenous infusion over 60 minutes, which can be reduced to 30 minutes if well tolerated.

\section{Clinical Efficacy and Limitations of Ramucirumab REACH and REACH-2 Trials}

The first published study to examine the efficacy and safety of ramucirumab monotherapy in advanced HCC was 
Table I Completed and Ongoing Trials Involving Ramucirumab in Hepatocellular Carcinoma

\begin{tabular}{|c|c|c|c|c|c|}
\hline Study (NCT) & HCC Population (n) & $\operatorname{Arm}(s)$ & $\begin{array}{c}\text { Primary } \\
\text { Outcome(s) }\end{array}$ & $\begin{array}{l}\text { Secondary } \\
\text { Outcome(s) }\end{array}$ & Results \\
\hline $\begin{array}{l}\text { Zhu et al, 2013 } \\
\text { (NCT00627042) }\end{array}$ & $\begin{array}{l}\text { Unresectable, untreated } \\
\text { HCC (42) }\end{array}$ & Ramucirumab & PFS & ORR, OS & $\begin{array}{l}\text { mPFS: } 4.0 \text { months ( } 95 \% \mathrm{Cl}: 2.6-5.7) \text { ORR: } 9.5 \%(95 \% \mathrm{Cl} \text { : } \\
2.7-22.6 ; 4 / 42 \text { PR) } \\
\text { mOS: } 12.0 \text { months ( } 95 \% \mathrm{Cl}: 6.1-19.7)\end{array}$ \\
\hline $\begin{array}{l}\text { Zhu et al, 2015 } 5^{17} \\
\text { (NCTOI 140347) }\end{array}$ & $\begin{array}{c}\text { Unresectable, 2nd line } \\
\text { HCC (565) }\end{array}$ & $\begin{array}{l}\text { 1) Ramucirumab + } \\
\text { BSC (283) } \\
\text { 2) placebo + BSC } \\
\text { (282) }\end{array}$ & OS & PFS, ORR & $\begin{array}{l}\text { mOS: } 9.2 \text { months for Ramucirumab }(95 \% \mathrm{Cl}: 8.0-10.6) \text { vs } \\
7.6 \text { months for placebo }(95 \% \mathrm{Cl}: 6.0-9.3) \text {; } \mathrm{HR} 0.87 \\
(P=0.14) \\
\text { mPFS: } 2.8 \text { months for Ramucirumab }(95 \% \mathrm{Cl}: 2.7-3.9) \text { vs } \\
2.1 \text { months for placebo }(95 \% \mathrm{Cl}: 1.6-2.7) \\
\text { ORR: } 7 \%(95 \% \mathrm{Cl}: 4.6-10.7) \text { in the Ramucirumab } \\
\text { group vs } \\
<1 \% \text {; } 95 \% \mathrm{Cl}: 0.2-2.5) \text { in the placebo group. }\end{array}$ \\
\hline $\begin{array}{l}\text { Zhu et al, } 2019^{19} \\
\text { (NCT02435433) }\end{array}$ & $\begin{array}{l}\text { Unresectable, } 2 \text { nd line } \\
\text { HCC with AFP } \geq 400 \\
\text { ng/mL (292) }\end{array}$ & $\begin{array}{l}\text { 1) Ramucirumab + } \\
\text { BSC (197) } \\
\text { 2) placebo + BSC } \\
\text { (95) }\end{array}$ & OS & PFS, ORR & $\begin{array}{l}\text { mOS: } 8.5 \text { months for Ramucirumab }(95 \% \mathrm{Cl}: 7.0-10.6) \text { vs } \\
7.3 \text { months for placebo }(95 \% \mathrm{Cl}: 5.4-9.1) \text {; HR } 0.710 \\
(P=0.0199) \\
\text { mPFS: } 2.8 \text { months for Ramucirumab }(95 \% \mathrm{Cl}: 2.8-4.1) \text { vs } \\
\text { I.6 months for placebo ( } 95 \% \mathrm{Cl}: 1.5-2.7) \text {; HR } 0.452 \\
(P<0.000 \mathrm{I}) \\
\text { ORR: } 9 / 197 \text { (Ramucirumab) vs I/95 (placebo); } P=0.1697\end{array}$ \\
\hline $\begin{array}{l}\text { Lin et al, } 2020^{55} \\
\text { (NCT0206904I) }\end{array}$ & $\begin{array}{c}\text { Unresectable, untreated } \\
\text { (Ist line) HCC (8) }\end{array}$ & $\begin{array}{l}\text { Ramucirumab + } \\
\text { FOLFOX }\end{array}$ & SAEs & $\begin{array}{l}\text { PK, ORR } \\
\text { (CR or PR) }\end{array}$ & $\begin{array}{l}\text { SAEs: } 100 \%(8 / 8) \\
\text { DCR: }(C R+P R): 62.5 \% \\
\text { ORR: } 25.0 \%\end{array}$ \\
\hline $\begin{array}{l}\text { Harding et al, } \\
2019^{56} \\
(\text { NCT0208220) }\end{array}$ & Advanced HCC (45) & $\begin{array}{l}\text { Ramucirumab + } \\
\text { emibetuzumab } \\
\text { (anti-MET) }\end{array}$ & $\begin{array}{l}\text { ORR (PR, } \\
\quad C R)\end{array}$ & PK, DCR, PFS & $\begin{array}{l}\text { ORR: } 6.7 \% \\
\text { DCR: } 60 \% \\
\text { mPFS: } 5.42 \text { months ( } 8.1 \text { in high MET vs } 2.8 \text { in low MET } \\
\text { expression) }\end{array}$ \\
\hline $\begin{array}{l}\text { Harding et al, } \\
2021^{57} \\
\text { (NCTOI 246986) }\end{array}$ & $\begin{array}{c}\text { Unresectable, 2nd line } \\
\text { HCC (8) }\end{array}$ & $\begin{array}{l}\text { Ramucirumab + } \\
\text { Galunisertib (anti- } \\
\text { TGF- } \beta \text { ) }\end{array}$ & SAEs & N/A & SAEs: $3 / 8(37.5 \%)$ \\
\hline NCT02572687 & $\begin{array}{l}\text { Unresectable, 2nd line } \\
\text { HCC with AFP } \geq 1.5 x \\
\text { ULN }\end{array}$ & $\begin{array}{c}\text { Ramucirumab + } \\
\text { Durvalumab }\end{array}$ & DLT & $\begin{array}{l}\text { ORR, DCR, } \\
\text { DoR, TTR, } \\
\text { PFS, OS }\end{array}$ & $\mathrm{N} / \mathrm{A}$; ongoing trial \\
\hline
\end{tabular}

Abbreviations: NCT, National Clinical Trial Number; n, number of participants; HCC, Hepatocellular carcinoma; PFS, progression-free survival; ORR, Objective response rate; PR, Partial Response; OS, Overall survival; TRAEs, Treatment-related adverse events; m, median; 95\% Cl, 95\% confidence interval; BSC, Best support care; HR, Hazard Ratio; AFP, Alpha-fetoprotein; PK, Pharmacokinetics; Cmax, maximum concentration; AUC, Area under the Curve; CR, Complete response; SAE, Serious Adverse Event (grade $\geq 3$ ); NR, Not reported; N/A, not applicable; ULN, Upper limit of normal; DCR, Disease control rate; DLT, Dose-limit toxicity; DoR, Duration of Response; TTR, Time to first response.

a single-arm study involving untreated patients $(n=42)$ in the first-line setting. ${ }^{18}$ Results revealed a median progression free survival (PFS) of 4.0 months (95\% confidence interval-CI-: 2.6-5.7), an overall response rate (ORR) of 9.5\% (95\% CI: 2.7-22.6) with 4 patients exhibiting partial response, and a median overall survival (OS) of 12.0 months (95\% CI: 6.1-197.7). Survival benefit was more pronounced in patients with Barcelona Clinic Liver Cancer (BCLC) stage $\mathrm{C}$ and Child-Pugh A cirrhosis. While prior attempts involving anti-angiogenic agents (such as bevacizumab) did not demonstrate efficacy in HCC, this was the first Phase II study to demonstrate effective anti-cancer activity of an anti-VEGF monotherapy agent with an acceptable safety profile. Of note, while sorafenib tyrosine kinase inhibitors VEGF receptors among other tyrosine kinases, this study was initiated prior to the approval of sorafenib for 1 st line treatment of advanced HCC.

These positive results lead to the initiation of the Phase III REACH clinical trial ${ }^{19}$ that evaluated ramucirumab + best supportive care (BSC), compared to placebo $+\mathrm{BSC}$, in the second-line treatment of patients who previously received sorafenib. Despite manageable side effects, ramucirumab did not significantly improve median OS compared to placebo $(9.2$ vs 7.6 months) or median PFS (2.9 vs 2.1 months). Of note, 
a trend toward survival benefit was observed only in patients with Child-Pugh A cirrhosis, similar to earlier observations. ${ }^{20}$ However, a subgroup of analysis of patients with elevated of alpha-fetoprotein (AFP $\geq 400 \mathrm{ng} / \mathrm{mL}$ ) revealed a significantly higher efficacy for ramucirumab $(\mathrm{n}=119)$ compared to placebo $(\mathrm{n}=131)$ with mOS of 7.8 months (vs 4.2 months; HR $0.67 ; P<0.001$ ), and a mPFS of 2.7 months (vs 1.5 months; HR: 0.70).${ }^{19,20}$ Subsequently, the efficacy of ramucirumab was specifically evaluated as a second-line treatment after sorafenib in patients with advanced $\mathrm{HCC}$ and $\mathrm{AFP} \geq 400 \mathrm{ng} / \mathrm{mL}$, in a phase III randomized clinical trial (REACH-2; NCT 02435433). ${ }^{21}$ Overall, participants had an unfavorable prognosis as characterized by elevated AFP levels and by the high proportion of patients with extrahepatic disease ( $75 \%)$. Median OS was 8.5 months in patient who received ramucirumab (vs 7.3 months in placebo; HR 0.71; $P=0.0199$ ), and median PFS of 2.8 months for ramucirumab treatment arm (vs 1.6 months in placebo; HR 0.452; $P<0.0001$ ). This survival benefit (OS and PFS) of ramucirumab was confirmed via pooled analysis of patients with AFP $\geq 400 \mathrm{ng} / \mathrm{mL}$ from REACH and REACH-2, with a mOS of 8.1 months (vs 5.0 months in placebo; HR $0.69 ; P=0.0002$ ) and mPFS of 2.8 months (vs 1.5 months for placebo; $P<0.0001$ ). ${ }^{22,23}$ Table 2 summarizes the survival benefit and effect on tumor response of ramucirumab in patients with an AFP $\geq 400 \mathrm{ng} / \mathrm{mL}$. Based on these results, the US FDA approved ramucirumab for treatment of HCC patients with an AFP $\geq 400 \mathrm{ng} / \mathrm{mL}$, who had previously been treated with sorafenib. ${ }^{24}$

\section{Real-World Data}

Since its FDA approval, several real-world studies have reported the therapeutic efficacy and safety of ramucirumab. ${ }^{25-29}$ In a study by Kuzuya et al, 12 patients were treated with ramucirumab after lenvatinib failure with a reported disease control rate (DCR) of $80 \%$ at 6 weeks and a median time to progression of 3.1 months. ${ }^{26}$ In a smaller study, 7 patients with unresectable HCC and AFP $\geq 400 \mathrm{ng} / \mathrm{mL}$ and previously treated with lenvatinib, received ramucirumab and achieved a DCR of $28.6 \%$ and a mPFS of 41 days. ${ }^{27}$ A recent similar study with a larger sample size $(n=28)$ reported a DCR of $42.3 \%$ and a mPFS of 2.0 months following failure of lenvatinib. ${ }^{28}$ In a study by Hatanaka et al exploring the role of albumin-bilirubin score in predicting outcome of ramucirumab for treatment of patients with advanced HCC, the treatment response of 16 patients was reported: ${ }^{29} \mathrm{DCR}$ ranged between $28.6 \%$ and $100 \%$, mOS ranged between 3.0 months and 6.7 months, and mPFS ranged between 1.4 and 7.5 months (depending on albumin-bilirubin score).

Despite relatively small sample sizes, these studies revealed similar to better survival and response benefit in the real-world compared to data from REACH trials. This is of special significance given the marginal survival benefit reported in trial data, as well as the restricted patient population for whom ramucirumab is approved.

\section{Ramucirumab Efficacy Across Patients' Subgroups (Table 3)}

Serum Alpha-Fetoprotein Levels

Besides demonstrating the clinical efficacy of the first-angiogenic agent monotherapy in advanced HCC, the REACH-2 trial was also the first positive biomarker-driven study in this patient population based on AFP levels. Elevated AFP is associated with worse prognosis compared to the general population, and incremental changes in AFP levels at the

Table 2 Ramucirumab Efficacy in Patients with Advanced Hepatocellular Carcinoma and $\alpha$-Fetoprotein Levels $\geq 400 \mathrm{ng} / \mathrm{mL}$ Treated Following Failure of First-Line Sorafenib: Data from Randomized, Double-Blind Phase 3 Trials

\begin{tabular}{|c|c|c|c|c|c|c|c|}
\hline \multirow[t]{2}{*}{ Study } & \multirow[t]{2}{*}{ Treatment (n) } & \multicolumn{2}{|c|}{ os } & \multicolumn{2}{|c|}{ PFS } & \multirow{2}{*}{$\begin{array}{c}\text { DCR } \\
(\%)\end{array}$} & \multirow[t]{2}{*}{ Reference } \\
\hline & & Months & HR & Months & HR & & \\
\hline \multirow[t]{2}{*}{ REACH $^{a}$} & Ramucirumab (1 19) & 7.8 & $0.67^{*}$ & 2.7 & 0.70 & & 17 \\
\hline & Placebo (I3I) & 4.2 & & 1.5 & & & \\
\hline \multirow[t]{2}{*}{ REACH-2 } & Ramucirumab (197) & 8.5 & $0.710 *$ & 2.8 & $0.452 * * *$ & $59.9 * *$ & 18 \\
\hline & Placebo (95) & 7.3 & & 1.6 & & 38.9 & \\
\hline \multirow[t]{2}{*}{ Pooled analysis } & Ramucirumab (316) & 8.1 & $0.694 * *$ & 2.8 & $0.572 * * *$ & $56.3 * * *$ & 18 \\
\hline & Placebo (226) & 5.0 & & 1.5 & & 37.2 & \\
\hline
\end{tabular}

Notes: ${ }^{a} \alpha$-fetoprotein $\geq 400 \mathrm{ng} / \mathrm{mL}$ subgroup; $* \mathrm{p}<0.0$ I; $* * \mathrm{p}<0.001$; $* * * P<0.0001$.

Abbreviations: OS, Overall survival; PFS, progression-free survival; DCR, Disease control rate; HR, Hazard Ratio. 
Table 3 Summary of Subgroup Analyses of Pooled Data from REACH and REACH 2 for Patients with $\alpha$-Fetoprotein Levels $\geq 400 \mathrm{ng} / \mathrm{mL}$

\begin{tabular}{|c|c|c|c|c|c|c|}
\hline \multirow[t]{2}{*}{ Subgroup } & \multicolumn{2}{|c|}{ Number } & \multicolumn{3}{|c|}{ Ramucirumab vs Placebo } & \multirow[t]{2}{*}{$\mathbf{R f}$} \\
\hline & RAM & PL & OS (Months; HR; 95\% Cl) & PFS (Months; HR; 95\% Cl) & DCR (\%) & \\
\hline \multicolumn{7}{|l|}{ Race } \\
\hline Asian & 168 & 123 & 8.1 vs $4.8(0.73 ; 0.56-0.95)$ & 2.7 vs I.5 $(0.58 ; 0.44-0.76)$ & 53.6 vs 33.3 & 29 \\
\hline Non-Asian & 148 & 103 & 8.0 vs $5.2(0.65 ; 0.49-0.86)$ & 3.1 vs $I .9(0.55 ; 0.4 I-0.73)$ & 59.5 vs 41.7 & 29 \\
\hline Japanese & 61 & 40 & 10.8 vs $4.5(0.56 ; 0.35-0.89)$ & 3.9 vs I.4 $(0.34 ; 0.21-0.55)$ & 67.2 vs 35.0 & 30 \\
\hline Age & & & & & & 31 \\
\hline$<65$ years & 171 & 131 & 8.2 vs $4.8(0.72 ; 0.56-0.92)$ & 2.7 vs I. $(0.62 ; 0.48-0.79)$ & N/A & \\
\hline$\geq 65$ to $<75$ years & 93 & 37 & 7.6 vs $5.2(0.59 ; 0.4 I-0.85)$ & 2.8 vs I.8 $(0.55 ; 0.39-0.78)$ & N/A & \\
\hline$\geq 75$ years & 52 & 28 & 8.9 vs $6.3(0.64 ; 0.39-1.1)$ & 4.2 vs I.6 $(0.48 ; 0.29-0.78)$ & N/A & \\
\hline HCC etiology & & & & & & 28 \\
\hline HBV & 124 & 101 & 7.7 vs $4.5(0.74 ; 0.55-0.99)$ & 2.7 vs I. $(0.55 ; 0.41-0.74)$ & 53.2 vs 28.7 & \\
\hline $\mathrm{HCV}$ & 76 & 51 & 8.2 vs $5.5(0.82 ; 0.55-1.23)$ & 3.6 vs $2.7(0.58 ; 0.39-0.88)$ & 65.8 vs 52.9 & \\
\hline Other & 116 & 74 & 8.5 vs $5.4(0.56 ; 0.40-0.79)$ & 2.8 vs $1.6(0.57 ; 0.41-0.79)$ & 53.4 vs 37.8 & \\
\hline Liver disease stage & & & & & & 33 \\
\hline BCLC-stage $B$ & 30 & 22 & 13.7 vs $8.2(0.43 ; 0.23-0.82)$ & 4.2 vs $2.8(0.33 ; 0.17 ; 0.64)$ & 80 vs 59 & \\
\hline BCLC-stage $C$ & 286 & 204 & 7.7 vs $4.8(0.72 ; 0.59-0.89)$ & 2.8 vs $1.5(0.60 ; 0.49-0.74)$ & 54 vs 35 & \\
\hline Prior TACE & & & & & & 34 \\
\hline Yes & 179 & 123 & 8.2 vs $5.2(0.69 ; 0.53-0.89)$ & 2.8 vs $1.5(0.56 ; 0.43-0.72)$ & 56 vs 36 & \\
\hline No & 137 & 103 & 7.7 vs $5.0(0.71 ; 0.52-0.95)$ & 2.8 vs $1.6(0.58 ; 0.43-0.79)$ & 56 vs 39 & \\
\hline $\begin{array}{l}\text { Sorafenib } \\
\text { discontinuation }\end{array}$ & & & & & & 35 \\
\hline Intolerance & 42 & 28 & 10.2 vs $6.7(0.59 ; 0.34-1.02)$ & 4.4 vs I.4 $(0.32 ; 0.19-0.55)$ & & \\
\hline Disease progression & 274 & 198 & 8.0 vs $4.7(0.71 ; 0.58-0.88)$ & 2.7 vs I.6 $(0.64 ; 0.52-0.79)$ & & \\
\hline
\end{tabular}

Abbreviations: RAM, Ramucirumab; PL, placebo; OS, Overall survival; PFS, Progression-free survival; DCR, Disease control rate; $\mathrm{HR}$, Hazard ratio; $\mathrm{Cl}$, confidence interval; N/A, not available; HBV, hepatitis B; HCV, hepatitis C; BCLC, Barcelona Clinic Liver Cancer staging; TACE, Transarterial chemoembolization; Rf, reference.

time of HCC diagnosis are significantly associated with increased mortality. ${ }^{30,31}$ Additionally, serum AFP levels predict the risk of tumor recurrence following resection, ${ }^{32}$ and levels $\geq 400 \mathrm{ng} / \mathrm{mL}$ consistently predict poorer prognosis. ${ }^{33,34}$ This prognostic and predictive value of baseline AFP was further confirmed by a recent post-hoc analyses of clinical outcomes by AFP during, of both REACH and REACH-2 trials: ${ }^{23}$ baseline AFP was confirmed as a continuous and dichotomous ( $\geq 400 \mathrm{vs}<400 \mathrm{ng} / \mathrm{mL}$ ) prognostic factor that was predictive of survival benefit with ramucirumab. Similarly, ramucirumab was favored in terms of time to AFP progression, and survival was longer in patients with an AFP response compared to those without it. This study consolidated the role of serum AFP levels as a selection criterion and biomarker of treatment response to ramucirumab.

\section{HCC Etiology}

The impact of HCC etiology on response to ramucirumab was evaluated in a meta-analysis of data from REACH/REACH-2 trials involving only patients with AFP $\geq 400 \mathrm{ng} / \mathrm{mL} .{ }^{35}$ Evaluated etiologies included: hepatitis B ( $\mathrm{HBV} ; \mathrm{n}=225)$, hepatitis $\mathrm{C}(\mathrm{HCV} ; \mathrm{n}=127)$ or other etiology (significant alcohol use, steatohepatitis, hemochromatosis, unknown etiology; $n=190$ ). Although patients with chronic viral hepatitis were eligible in both studies, the impact of pre-treatment serum HBV DNA and HCV RNA on response to 
ramucirumab was also evaluated. The study revealed no significant difference in treatment effect by etiology subgroup: mOS (ramucirumab vs placebo) was 7.7 vs 4.5 months (HR 0.74; 95\% CI 0.55-0.99) for HBV, 8.2 vs 5.5 months (HR: 0.82 ; $95 \%$ CI $0.55-1.23$ ) for $\mathrm{HCV}$, and 8.5 vs 5.4 months (HR: 0.56 ; 95\% CI 0.40-0.79) for other. Similar results were seen in terms of mPFS and disease DCR (Table 3). The safety profile of ramucirumab was also compared across subgroups: although similar overall safety profiles were observed across the different etiologies, worst outcomes were seen in patients with a detectable HBV viral load, with use of antiviral therapy showing benefit for survival, liver function and liver-specific adverse events, irrespective of the viral load. ${ }^{35}$

\section{Other Subgroup Analyses}

Two pooled exploratory subgroup analyses were done comparing ramucirumab treatment benefit in Asian vs non-Asian patients, ${ }^{36}$ and in a subgroup of Japanese patients. $^{37}$ Benefits of ramucirumab were consistent with those of the overall population regardless of the race. Furthermore, ramucirumab treatment benefits were observed regardless of patient age, ${ }^{38,39}$ liver disease stage, ${ }^{40}$ prior transarterial chemoembolization (TACE), ${ }^{41}$ and reason for sorafenib discontinuation. ${ }^{42}$ These are summarized in Table 3.

\section{Limitations of Ramucirumab Therapy}

Despite the survival benefit of ramucirumab and its subsequent FDA approval for second-line therapy of patients with advanced $\mathrm{HCC}$ and $\mathrm{AFP} \geq 400 \mathrm{ng} / \mathrm{mL}$ who received 1 st line sorafenib, rates of disease progression remain significant for those who receive ramucirumab: $68.9 \%$ (vs 77.7\% for placebo) in the REACH subgroup of patients with AFP $\geq 400 \mathrm{ng} / \mathrm{mL}$, and $70 \%$ (vs $76.8 \%$ for placebo) in the REACH-2 trial. ${ }^{19,21,43}$ Pooled analysis of 625 patients from both REACH and REACH-2 trials, who showed radiological progression examined possible association between patterns of progression (extrahepatic lesion, intra-hepatic lesion, extrahepatic growth or intrahepatic growth). ${ }^{43}$ While ramucirumab provided an overall survival benefit across different progression patterns, OS was significantly reduced in patients with new extrahepatic lesions (HR 2.33 and 1.49 in REACH and REACH-2, respectively, and HR 1.75 in pooled analysis), conferring poor prognosis for post-progression survival.

Clinically, finding from both trials including rates of disease progression translate into a very modest absolute gain in medial survival of 1.2 months only, raising questions about the practical benefit, namely in light of associated costs and toxicity. ${ }^{44}$ This marginal survival benefit was attributed to the fact that long median OS was also reached by the placebo group, ${ }^{21}$ further raising questions about the interpretation of AFP and its prognostic value in patient selection for treatment for ramucirumab. Another challenge in translating the study findings into daily clinical practice pertains to the exclusion of patients at high risk of bleeding associated with liver disease (high-risk varices). Similarly, a clinical limitation of ramucirumab pertains to its selective approval for use in patients with Child-Pugh class A only, with no available safety data in patients with Child-Pugh B liver function to support its use in this patient's population.

On a molecular level, the relationship between AFP levels and tumor response remains poorly understood, and the interpretation of a potential relationship between elevated AFP levels (indicative of poor prognosis/advanced disease) and the tumor molecular biology that favors response to an anti-angiogenic agent like ramucirumab remains to be explored. Whether elevated AFP as surrogate markers of an advanced tumor could also reflect a higher immunogenicity remain to be explored and could potentially justify combining ramucirumab with immune therapies. ${ }^{45}$

\section{Tolerability}

The three main trials evaluating the use of intravenous ramucirumab ( $8 \mathrm{mg} / \mathrm{kg}$, every 2 weeks) in patients with $\mathrm{HCC}$, revealed acceptable tolerability and a manageable safety profile. ${ }^{18,19,21}$ In the HCC population with current FDA indication to use ramucirumab (AFP $\geq 400 \mathrm{ng} / \mathrm{mL}$ ), safety and tolerability data are available from the REACH2 and the pooled analysis of REACH and REACH-2 ( $n=$ 316 for ramucirumab and $\mathrm{n}=233$ for placebo). ${ }^{19,21}$

In the pooled analysis, $9.5 \%$ of patients who received ramucirumab had a treatment-related adverse event (TRAE) compared to $3.6 \%$ in the placebo group. The most common TRAEs (of any grade) with ramucirumab included: nausea, fatigue, anorexia, peripheral edema, diarrhea, headache and abdominal pain. Most of these were mild/moderate TRAEs except for 2 patients who had grade $\geq 3$ peripheral edema, 2 patients with grade $\geq 3$ fatigues, and 1 patient with abdominal pain. In terms of treatment-emergent AEs, the only grade $\geq 3$ events that occurred in $\geq 5 \%$ of patients treated with ramucirumab were hypertension ( $13 \%$ vs $4 \%$ in placebo) and hyponatremia ( $5 \%$ vs $2 \%$ in placebo). ${ }^{19,21}$ 
In REACH-2 ( $\mathrm{n}=197$ for ramucirumab and $\mathrm{n}=95$ for placebo), TRAEs of any grade occurred in $11 \%$ of patients treated with ramucirumab, compared to $5 \%$ in placebo recipient. ${ }^{21}$ Hepatic encephalopathy and dyspnea occurred only in the ramucirumab group. All-cause death rates ( $20 \%$ vs $17 \%$ for placebo), and on-treatment death due to AEs ( $3 \%$ in each group), were similar across both groups. In the ramucirumab group, 3 deaths (from acute kidney injury, hepatorenal syndrome, and renal failure) were deemed to be treatment-related. Compared to tyrosine kinase inhibitors such as sorafenib, ramucirumab showed a more favorable toxicity profile. ${ }^{46}$ A detailed guide for the management of ramucirumab's most common treatment related side effects has already been published. ${ }^{47}$

As pre-specified by the study, treatment-related adverse events of special interest (AESIs) included: hypertension, proteinuria, bleeding/hemorrhage, liver injury/failure, and infusion-related reactions (IRRs) that occur within 24 hours of infusion. ${ }^{21}$ In the pooled analysis, AESIs of any grade were more common with ramucirumab compared to placebo. In terms of grade $\geq 3$ AESIs, hypertension occurred more frequently with ramucirumab $(8.2 \%$ vs $1.3 \%$ in placebo), and liver injury or failure occurred in $4.7 \%$ and $4.5 \%$ of ramucirumab and placebo recipients. All other ASEIs occurred at similar frequency and in $\leq 2 \%$ in both groups. IRRs to ramucirumab were observed during or following the first of second infusion leading to the recommendation to pre-medicate with anti-histamine, dexamethasone and paracetamol prior to ramucirumab infusion, to decrease both incidence and severity of IRRs. ${ }^{48}$ Other reported AESI related to ramucirumab included: gastrointestinal perforation, thromboembolic events, impaired wound health, posterior reversible encephalopathy syndrome, thyroid dysfunction, and embryo-fetal toxicity. ${ }^{24,48}$ Whether the different adverse events of ramucirumab carry any significant prognostic significance remains unknown: in fact, multiple prior retrospective studies and post-hoc analyses of clinical trials of sorafenib in HCC suggested a prognostic significance of adverse events in terms of prolonged time to progression and longer OS. ${ }^{49}$ These adverse events included dermatologic toxicities, ${ }^{50,51}$ hypertension, ${ }^{52}$ and diarrhea. ${ }^{53,54}$ Given these reports, albeit retrospective, along with the similar toxicity profile, future studies ought to explore any potential correlation between ramucirumab associated toxicities and treatment response.

In terms of quality of life (QoL) and performance status (PS), analysis of patient-focused outcomes from
REACH trial using the FACT Hepatobiliary Symptom Index (FHSI)-8 and the EuroQoL (EQ-5D), revealed a significantly reduced deterioration in FHSI-8 in patients with AFP $400 \mathrm{ng} / \mathrm{mL}$ who received ramucirumab compared to placebo. ${ }^{55}$ Similarly, a trend towards a delay in symptomatic deterioration (using FHSI- $8, P=0.054$ and PS, $P=0.057$ ) in favor of ramucirumab. In the non-AFP selected population, ramucirumab was associated with no worsening of QoL compared to placebo.

\section{Ramucirumab and Therapeutic Strategies for the Treatment of Hepatocellular Carcinoma}

\section{Ramucirumab and Current Management of HCC}

Currently, ramucirumab is the only therapy specifically tested in a biomarker (AFP)-selected HCC population, with a recommendation only for patients with AFP $\geq$ $400 \mathrm{ng} / \mathrm{mL}^{11,42,43}$ Other available molecular targeted systemic therapies for advanced HCC include sorafenib, lenvatinib, regorafenib, and cabozantinib, all of which are multikinase inhibitors, and more recently the combination of atezolizumab and bevacizumab. While bevacizumab targets VEGF, ramucirumab is the only VEGF receptor (VEGFR-2) antagonist approved for HCC. Other therapies include immune checkpoint inhibitors: while nivolumab was originally indicated based on response data from a Phase $1 / 2$ trial, recent Phase 3 trial data did not support the use of single-agent nivolumab in the second-line setting of advanced HCC that failed first-line sorafenib. ${ }^{58}$ This is in contrast to the recent FDA accelerated approval of pembrolizumab monotherapy for patients with advanced HCC who received prior treatment with sorafenib, based on data from KEYNOTE-240 and KEYNOTE-394. ${ }^{59}$

Sorafenib had long been the standard first-line systemic therapy for patients with advanced HCC and well-reserved liver function, and those unsuitable for locoregional therapies. ${ }^{11,42,43}$ However, the recent data from IMbrave 150 phase 3 trial showed that the combination of atezolizumab plus bevacizumab had significantly longer 12 month OS $(67.2 \%$ vs $54.6 \%$; HR $0.58, P<0.001)$ and median PFS (6.8 vs 4.3 months; HR 0.59; $P<0.001$ ) compared with sorafenib, making the combination the preferred first-line treatment of patients with advanced HCC. ${ }^{60-62}$ Lenvatinib is another first-line option in these patients. Regorafenib had been the standard second-line 
systemic therapy following progression on sorafenib, until recent guidelines also recommended cabozantinib and ramucirumab. Other recommended second-line regimens include nivolumab plus Ipilimumab combination, and pembrolizumab monotherapy. ${ }^{12}$

\section{Ongoing Evaluation of Ramucirumab-Based Combinational Treatment Strategies}

Combining targeted therapies designed to inhibit specific oncogenic pathways, such as ramucirumab, with systemic chemotherapy or immune checkpoint inhibitors has been an attractive strategy to harness and maximize anti-tumor effects with acceptable side effects. ${ }^{63,64}$ For agents targeting VEGF or its receptors, the rationale is related to the potential of those agents to normalize tumor vasculature and contribute to increased T-cell infiltration, as well as promoting dendritic cell maturation and inhibition of immunosuppressive immune cells like regulatory T-cells and myeloid-derived suppressor cells. ${ }^{65-67}$ There is thus good promise in combining VEGF-targeting therapies with anti-PD-1/PD-L1 therapy with considerable synergistic antitumor activity shown in Phase I to III, notably those involving bevacizumab (anti-VEGF antibody). ${ }^{66}$ Clinically, for patients with advanced HCC bevacizumab administered in combination with atezolizumab significantly improved OS and PFS compared to sorafenib in the first-line setting (HR $0.58 ; 95 \%$ CI $0.42-0.79 ; P<0.001) .{ }^{60}$ The recent approval of atezolizumab in combination with bevacizumab in the first-line setting treatment of patients with advanced HCC thus opens the way for trials that evaluate ramucirumab in combination with checkpoint inhibitors. ${ }^{68}$

In fact, combination of ramucirumab with anti-PD1 pembrolizumab or with anti-PD-L1 durvalumab in previously treated advanced gastrointestinal cancers, nonsmall cell lung cancer, and urothelial carcinoma has shown a favorable anti-tumor activity with a manageable safety profile. ${ }^{69-71}$ Currently, one ongoing trial in HCC specifically (NCT02572687) is evaluating combination ramucirumab with durvalumab in unresectable $\mathrm{HCC}$ with AFP 1.5x upper limit of normal who failed sorafenib (Table 1). The primary outcome of the study is dose-limiting toxicity with secondary outcomes including ORR, DCR, OS, PFS and duration of response.

Ramucirumab has also been evaluated in combination with chemotherapy: in a non-randomized, phase Ib trial, a safety cohort of 8 patients with unresectable, previously untreated HCC combination ramucirumab with FOLFOX chemotherapy revealed $62.5 \%$ disease control rate (complete and partial response) and a $25 \%$ overall response rate (including stable disease); however, $100 \%$ of patients exhibited serious adverse events (Table 1$)^{72}$

Few studies have evaluated the feasibility of combining ramucirumab with other molecularly targeted agents in the treatment of HCC. In a phase Ib/II trial (NCT0208220) involving patients with advanced solid tumors, combination of emibetuzumab -a monoclonal antibody targeting MET- with ramucirumab was evaluated in a cohort of 45 patients with advanced HCC: ${ }^{73}$ the study revealed no dose-limiting toxicities, and adverse events were mild or moderate. While the combination revealed partial response $(5.2 \%)$ across different solid tumors $(n=97)$, the greatest antitumor activity was noted in HCC: ORR was $6.7 \%$ with $60 \%$ DCR and 5.42 months of PFS. ${ }^{73}$ Interestingly, HCC patients with high MET expression showed improved PFS relative low MET expression (8.1 months vs 2.8 months respectively). Beyond the safety and potential efficacy of combined ramucirumab-based targeted therapy, the study highlighted the potential for signal-based treatment selection when combining therapies with ramucirumab. Another recently published phase Ib (NCT01246986) study investigated the combination of ramucirumab with galunisertib, an inhibitor of TGF- $\beta$ receptor. ${ }^{74}$ The rationale behind the combination is based upon the known interaction between VEGF and TGF- $\beta$ pathways, which stimulates angiogenesis and suppress antitumor immune response in HCC. In this study, 8 patients with advanced HCC received the combination: while no dose-limiting toxicities were observed and the combination was overall well tolerated, the combination revealed very modest antitumor effect with $0 \%$ ORR and $12.5 \%$ DCR. Table 1 summarizes all completed and ongoing trial involving ramucirumab in patients with advanced HCC.

\section{Conclusions and Prospects}

Ramucirumab prolongs survival of advanced HCC in the second-line setting and has an overall tolerable and manageable safety profile. Currently, it is indicated only for patients with AFP $\geq 400 \mathrm{ng} / \mathrm{mL}$. While survival advantage is pronounced in this molecular group, the biological relationship between higher level of AFP and this significant response to therapy remains not fully understood. Other considerations also remain to be addressed namely about the practical implications of a modest absolute gain of survival (median 1.2 months), and how to balance this 
with the drugs cost and side effects. There is thus a need for further clinical data to assess the efficacy of ramucirumab in a broader patient population, and possibly within a combinatorial therapeutic approach. Similarly, clinical data is needed for a head-to-head comparison of ramucirumab and other agents in the second-line setting of advanced HCC. Future research ought to delineate safe and successful ramucirumab-based combinations such as other small targeted molecules and TKIs, as well as immune checkpoint inhibitors, including PD-1/PD-L1 inhibitors and others, especially in light of the recent approval of atezolizumab in combination with bevacizu$\mathrm{mab}$ in the first-line setting treatment of patients with advanced HCC. The recently announced overall positive results from the HIMALAYA Phase 3 trial further highlights the potential impact of immune checkpoint inhibitors in the treatment of advanced HCC, whereby combination of a one priming dose of tremelimumab (CTLA-4 inhibitor) plus durvalumab demonstrated statistically significant and clinically meaningful OS benefit compared to sorafenib in the first-line treatment of patients with unresectable, treatment-naïve HCC. $^{75}$ From the perspective of ramucirumab, the upcoming likely approval of this regimen in the frontline setting will strengthen the position of ramucirumab as a viable later line option for patients with high AFP.

\section{Abbreviations}

HCC, Hepatocellular carcinoma; AFP, alpha-fetoprotein; BCLC, Barcelona Staging Classification; TNM, Tumor/ Node/Metastasis; TKI, tyrosine kinase inhibitor; PFS, progression free survival; ORR, overall response rate; OS, overall survival; BSC, best supportive care; HBV, hepatitis $\mathrm{B}$; HCV, hepatitis $\mathrm{C}$; DCR, disease control rate; TACE, transarterial chemoembolization; TRAE, treatment-related adverse event; AESIs, adverse events of special interest; IRRs, infusion-related reactions; QoL, quality of life; PS, performance status; FHSI-8, FACT Hepatobiliary Symptom Index; EQ-5D, EuroQoL.

\section{Author Contributions}

$\mathrm{AS}$ and $\mathrm{KC}$ conceived and designed the structure of this manuscript. $\mathrm{KC}$ and SK reviewed the literature and collated data from major studies and clinical trials. $\mathrm{KC}$ and SK provided the initial draft of the manuscript, including the tables. $\mathrm{AS}, \mathrm{KC}$ and $\mathrm{SK}$ revised the manuscript for important intellectual content. All authors contributed to data analysis, drafting and revising of the article, have agreed on the journal to which the article will be submitted, gave final approval of the version to be published, and agree to be accountable for all aspects of the work.

\section{Funding}

No funding was received.

\section{Disclosure}

AS reports research grants (to institution) for unrelated research work from AstraZeneca, Bristol Myers Squibb, Merck, Exelixis and Clovis and Advisory board/consultant fees from AstraZeneca, Bristol Myers Squibb, Merck and Pfizer. The remaining authors declare that they have no competing interests.

\section{References}

1. Key statistics about liver cancer. Available from: https://www.cancer. org/cancer/liver-cancer/about/what-is-key-statistics.html. Accessed October 9, 2021.

2. Villanueva A. Hepatocellular Carcinoma. N Engl J Med. 2019;380 (15):1450-1462. doi:10.1056/NEJMra1713263

3. Kulik L, El-Serag HB. Epidemiology and management of hepatocellular carcinoma. Gastroenterology. 2019;156(2):477-491.e1. doi:10.1053/j.gastro.2018.08.065

4. Bialecki ES, Di Bisceglie AM. Diagnosis of hepatocellular carcinoma. HPB (Oxford). 2005;7(1):26-34. doi:10.1080/ 13651820410024049

5. Ryder SD. British Society of Gastroenterology. Guidelines for the diagnosis and treatment of hepatocellular carcinoma (HCC) in adults. Gut. 2003;52 Suppl 3:iii1-8. doi:10.1136/gut.52.suppl_3.iii1

6. Llovet JM, Brú C, Bruix J. Prognosis of hepatocellular carcinoma: the BCLC staging classification. Semin Liver Dis. 1999;19 (3):329-338. doi:10.1055/s-2007-1007122

7. Marrero JA, Fontana RJ, Barrat A, et al. Prognosis of hepatocellular carcinoma: comparison of 7 staging systems in an American cohort. Hepatology. 2005;41(4):707-716. doi:10.1002/hep.20636

8. Cillo U, Vitale A, Grigoletto F, et al. Prospective validation of the Barcelona Clinic Liver Cancer staging system. J Hepatol. 2006;44 (4):723-731. doi:10.1016/j.jhep.2005.12.015

9. Lobo L, Yakoub D, Picado O, et al. Unresectable hepatocellular carcinoma: radioembolization versus chemoembolization: a systematic review and meta-analysis. Cardiovasc Intervent Radiol. 2016;39(11):1580-1588. doi:10.1007/s00270-016-1426-y

10. National Comprehensive Cancer Network. NCCN clinical practice guidelines in oncology: hepatobiliary cancers (version 3.2019); 2019. Available from: https://www.nccn.org. Accessed 2 Oct 2021.

11. Benson AB, D'Angelica MI, Abbott DE, et al. Hepatobiliary Cancers, Version 2.2021, NCCN Clinical Practice Guidelines in Oncology. J Nat Comprehen Cancer Network. 2021;19(5):541-565. doi:10.6004/jncen.2021.0022

12. National Comprehensive Cancer Network. NCCN clinical practice guidelines in oncology: hepatobiliary cancers (version 5.2021). Available from: https://www.nccn.org/guidelines/guidelines-detail? category=1\&id=1438. Accessed October 2, 2021.

13. Zhu AX, Finn RS, Mulcahy M, et al. A phase II and biomarker study of ramucirumab, a human monoclonal antibody targeting the VEGF receptor-2, as first-line monotherapy in patients with advanced hepatocellular cancer. Clin Cancer Res. 2013;19(23):6614-6623. doi:10.1158/1078-0432.CCR-13-1442 
14. Lilly E. Cyramza (ramucirumab) injection, for intravenous use: US prescribing information; 2019. Available from: https://www.cyramza. com/. Accessed December 22, 2021.

15. Granito A, Forgione A, Marinelli S, et al. Experience with regorafenib in the treatment of hepatocellular carcinoma. Therap Adv Gastroenterol. 2021;14:175628482110169. doi:10.1177/17562848211016959

16. Syed YY. Ramucirumab: a review in hepatocellular carcinoma. Drugs. 2020;80(3):315-322. doi:10.1007/s40265-020-01263-6

17. Spratlin JL, Cohen RB, Eadens M, et al. Phase I pharmacologic and biologic study of ramucirumab (IMC-1121B), a fully human immunoglobulin G1 monoclonal antibody targeting the vascular endothelial growth factor receptor-2. J Clin Oncol. 2010;28(5):780-787. doi:10.1200/JCO.2009.23.7537

18. Zhu AX, Finn RS, Mulcahy M, et al. A Phase II and Biomarker Study of Ramucirumab, a Human Monoclonal Antibody Targeting the VEGF Receptor-2, as First-Line Monotherapy in Patients with Advanced Hepatocellular Cancer. Clin Cancer Res. 2013;19 (23):6614-6623. doi:10.1158/1078-0432.CCR-13-1442

19. Zhu AX, Park JO, Ryoo BY, et al. Ramucirumab versus placebo as second-line treatment in patients with advanced hepatocellular carcinoma following first-line therapy with sorafenib (REACH): a randomised, double-blind, multicentre, phase 3 trial. Lancet Oncol. 2015;16(7):859-870. doi:10.1016/S1470-2045(15)00050-9

20. Zhu AX, Baron AD, Malfertheiner P, et al. Ramucirumab as Second-Line Treatment in Patients With Advanced Hepatocellular Carcinoma: analysis of REACH Trial Results by Child-Pugh Score. JAMA Oncol. 2017;3(2):235. doi:10.1001/jamaoncol.2016.4115

21. Zhu AX, Kang YK, Yen CJ, et al. Ramucirumab after sorafenib in patients with advanced hepatocellular carcinoma and increased $\alpha$ fetoprotein concentrations (REACH-2): a randomised, double-blind, placebo-controlled, phase 3 trial. Lancet Oncol. 2019;20(2):282-296. doi:10.1016/S1470-2045(18)30937-9

22. Zhu A, Finn R, Galle P, et al. Ramucirumab as second-line treatment in patients with advanced hepatocellular carcinoma (HCC) and elevated alpha-fetoprotein (AFP) following first-line sorafenib: pooled efficacy and safety across two global randomized Phase 3 studies (REACH-2 and REACH). Ann Oncol. 2018;29:v122. doi:10.1093/ annonc/mdy 208

23. Zhu AX, Finn RS, Kang YK, et al. Serum alpha-fetoprotein and clinical outcomes in patients with advanced hepatocellular carcinoma treated with ramucirumab. Br J Cancer. 2021;124(8):1388-1397. doi:10.1038/s41416-021-01260-w

24. FDA approves ramucirumab for hepatocellular carcinoma |FDA. Available from: https://www.fda.gov/drugs/resources-informationapproved-drugs/fda-approves-ramucirumab-hepatocellular-carci noma. Accessed September 29, 2021.

25. Maesaka K, Sakamori R, Yamada R, et al. Efficacy of Ramucirumab Versus Sorafenib as Subsequent Treatment for Hepatocellular Carcinoma. Anticancer Res. 2021;41(4):2187-2192. doi:10.21873/anticanres.14993

26. Kuzuya $\mathrm{T}$, Ishigami $\mathrm{M}$, Ito $\mathrm{T}$, et al. Initial Experience of Ramucirumab Treatment After Lenvatinib Failure for Patients With Advanced Hepatocellular Carcinoma. Anticancer Res. 2020;40 (4):2089-2093. doi:10.21873/anticanres.14167

27. Kasuya K, Kawamura Y, Kobayashi M, et al. Efficacy and Safety of Ramucirumab in Patients with Unresectable Hepatocellular Carcinoma with Progression after Treatment with Lenvatinib. Intern Med. 2021;60(3):345-351. doi:10.2169/internalmedicine.5185-20

28. Hiraoka A, Kumada T, Tada T, et al. Therapeutic efficacy of ramucirumab after lenvatinib for post-progression treatment of unresectable hepatocellular carcinoma. Gastroenterol Rep. 2021;9 (2):133-138. doi:10.1093/gastro/goaa042

29. Hatanaka T, Naganuma A, Shibasaki M, et al. The Role of the Albumin-Bilirubin Score for Predicting the Outcomes in Japanese Patients with Advanced Hepatocellular Carcinoma Treated with Ramucirumab: a Real-World Study. Oncology. 2021;99(4):203-214. doi:10.1159/000511734
30. Tyson GL, Duan Z, Kramer JR, Davila JA, Richardson PA, ElSerag HB. Level of $\alpha$-Fetoprotein Predicts Mortality Among Patients With Hepatitis C-Related Hepatocellular Carcinoma. Clin Gastroenterol Hepatol. 2011;9(11):989-994. doi:10.1016/j. cgh.2011.07.026

31. Wang Y, Chen Y, Ge N, et al. Prognostic Significance of Alpha-fetoprotein Status in the Outcome of Hepatocellular Carcinoma after Treatment of Transarterial Chemoembolization. Ann Surg Oncol. 2012;19(11):3540-3546. doi:10.1245/s10434-0122368-5

32. Imamura $H$, Matsuyama $Y$, Tanaka E, et al. Risk factors contributing to early and late phase intrahepatic recurrence of hepatocellular carcinoma after hepatectomy. $J$ Hepatol. 2003;38(2):200-207. doi:10.1016/S0168-8278(02)00360-4

33. Tangkijvanich P, Anukulkarnkusol N, Suwangool P, et al. Clinical Characteristics and Prognosis of Hepatocellular Carcinoma: analysis Based on Serum Alpha-fetoprotein Levels. J Clin Gastroenterol. 2000;31(4):302-308. doi:10.1097/00004836-200012000-00007

34. Nakazawa T, Hidaka H, Takada J, et al. Early increase in $\alpha$-fetoprotein for predicting unfavorable clinical outcomes in patients with advanced hepatocellular carcinoma treated with sorafenib. Eur J Gastroenterol Hepatol. 2013;25(6):683-689. doi:10.1097/ MEG.0b013e32835d913b

35. Galle PR, Kudo M, Llovet JM, et al. Ramucirumab in patients with previously treated advanced hepatocellular carcinoma: impact of liver disease aetiology. Liver Int. 2021:liv.14994. doi:10.1111/liv.14994

36. Yen CJ, Kudo M, Lim HY, et al. Efficacy and Safety of Ramucirumab in Asian and Non-Asian Patients with Advanced Hepatocellular Carcinoma and Elevated Alpha-Fetoprotein: pooled Individual Data Analysis of Two Randomized Studies. Liver Cancer. 2020;9 (4):440-454. doi:10.1159/000506946

37. Kudo M, Okusaka T, Motomura K, et al. Ramucirumab as second-line treatment in patients with advanced hepatocellular carcinoma (HCC) and elevated alpha-fetoprotein (AFP) following first-line sorafenib: pooled efficacy and safety in Japanese patients across two global randomized phase III studies (REACH-2 and REACH). JCO. 2019;37 (4_suppl):320. doi:10.1200/JCO.2019.37.4_suppl.320

38. Kudo M, Galle PR, Motomura K, et al. Efficacy and safety of ramucirumab (RAM) for advanced hepatocellular carcinoma (HCC) with elevated alpha-fetoprotein (AFP) following first-line sorafenib across age subgroups in two global phase III trials (REACH and REACH-2). Ann Oncol. 2019;30:v292. doi:10.1093/annonc/mdz247.083

39. Kudo M, Galle PR, Llovet JM, et al. Ramucirumab in elderly patients with hepatocellular carcinoma and elevated alpha-fetoprotein after sorafenib in REACH and REACH-2. Liver Int. 2020;40 (8):2008-2020. doi:10.1111/liv.14462

40. Kudo M, Finn RS, Morimoto M, et al. Ramucirumab for Patients with Intermediate-Stage Hepatocellular Carcinoma and Elevated Alpha-Fetoprotein: pooled Results from Two Phase 3 Studies (REACH and REACH-2). Liver Cancer. 2021;10:1-10. doi:10.1159/000516605

41. Meyer T, Finn R, Kudo M, et al. Ramucirumab in advanced hepatocellular carcinoma and elevated alpha-fetoprotein following sorafenib: outcomes by prior transarterial chemoembolisation from two randomised, double-blind, placebo-controlled phase 3 studies (REACH-2 and REACH). Ann Oncol. 2019;30:iv133. doi:10.1093/ annonc/mdz154.020

42. Llovet JM, Yen CJ, Finn RS, et al. Ramucirumab (RAM) for sorafenib intolerant patients with hepatocellular carcinoma (HCC) and elevated baseline alpha fetoprotein (AFP): outcomes from two randomized phase 3 studies (REACH, REACH2). JCO. 2019;37 (15_suppl):4073. doi:10.1200/JCO.2019.37.15_suppl.4073

43. Reig M, Galle PR, Kudo M, et al. Pattern of progression in advanced hepatocellular carcinoma treated with ramucirumab. Liver Int. 2021;41:598. 
44. De Luca E, Marino D, Di Maio M. Ramucirumab, A Second-Line Option For Patients With Hepatocellular Carcinoma: a Review Of The Evidence. CMAR. 2020;12:3721-3729. doi:10.2147/CMAR.S216220

45. Breous E, Thimme R. Potential of immunotherapy for hepatocellular carcinoma. $J$ Hepatol. 2011;54(4):830-834. doi:10.1016/j. jhep.2010.10.013

46. Galle PR, Dufour JF, Peck-Radosavljevic M, Trojan J, Vogel A. Systemic therapy of advanced hepatocellular carcinoma. Future Oncol. 2021;17(10):1237-1251. doi:10.2217/fon-2020-0758

47. Winters AC, Bedier F, Saab S. Management of Side Effects of Systemic Therapies for Hepatocellular Carcinoma. Clin Liver Dis. 2020;24(4):755-769. doi:10.1016/j.cld.2020.07.008

48. Lilly E. CYRAMZA (ramucirumab) injection, for intravenous useInitial U.S. prescribing information; 2019. Available from: https://uspl.lilly. com/cyramza/cyramza.html\#pi. Accessed October 2, 2021.

49. Granito A, Marinelli S, Negrini G, Menetti S, Benevento F, Bolondi L. Prognostic significance of adverse events in patients with hepatocellular carcinoma treated with sorafenib. Therap Adv Gastroenterol. 2016;9(2):240-249. doi:10.1177/1756283X15618129

50. Reig M, Torres F, Rodriguez-Lope C, et al. Early dermatologic adverse events predict better outcome in $\mathrm{HCC}$ patients treated with sorafenib. J Hepatol. 2014;61(2):318-324. doi:10.1016/j.jhep.2014.03.030

51. Vincenzi B, Santini D, Russo A, et al. Early Skin Toxicity as a Predictive Factor for Tumor Control in Hepatocellular Carcinoma Patients Treated with Sorafenib. Oncologist. 2010;15(1):85-92. doi:10.1634/theoncologist.2009-0143

52. Hamnvik OPR, Choueiri TK, Turchin A, et al. Clinical risk factors for the development of hypertension in patients treated with inhibitors of the VEGF signaling pathway: hypertension With Anti-VEGF Therapies. Cancer. 2015;121(2):311-319. doi:10.1002/cncr.28972

53. Bettinger D, Schulthei $\beta$ M, Knüppel E, Thimme R, Blum HE, Spangenberg HC. Diarrhea predicts a positive response to sorafenib in patients with advanced hepatocellular carcinoma. Hepatology. 2012;56(2):789-790. doi:10.1002/hep.25637

54. Koschny R, Gotthardt D, Koehler C, Jaeger D, Stremmel W, Ganten TM. Diarrhea Is a Positive Outcome Predictor for Sorafenib Treatment of Advanced Hepatocellular Carcinoma. Oncology. 2013;84(1):6-13. doi:10.1159/000342425

55. Chau I, Peck-Radosavljevic M, Borg C, et al. Ramucirumab as second-line treatment in patients with advanced hepatocellular carcinoma following first-line therapy with sorafenib: patient-focused outcome results from the randomised phase III REACH study. Eur $J$ Cancer. 2017;81:17-25. doi:10.1016/j.ejca.2017.05.001

56. Vogel A, Cervantes A, Chau I, et al. Hepatocellular carcinoma: ESMO Clinical Practice Guidelines for diagnosis, treatment and follow-up. Ann Oncol. 2018;29:iv238-iv255. doi:10.1093/annonc/ mdy 308

57. Gordan JD, Kennedy EB, Abou-Alfa GK, et al. Systemic Therapy for Advanced Hepatocellular Carcinoma: ASCO Guideline. JCO. 2020;38(36):4317-4345. doi:10.1200/JCO.20.02672

58. FDA Panel Opposes Nivolumab for Second-line Advanced HCC. Accessed October 26., 2021. Available from: https://www.onclive.com/ view/fda-panel-opposes-nivolumab-for-second-line-advanced-hcc.

59. ODAC Unanimously Backs Pembrolizumab for Second-line Advanced HCC. Available from: https://www.onclive.com/view/ odac-unanimously-backs-pembrolizumab-for-second-line-advancedhcc. Accessed October 24, 2021.

60. Finn RS, Qin S, Ikeda M, et al. Atezolizumab plus Bevacizumab in Unresectable Hepatocellular Carcinoma. N Engl J Med. 2020;382 (20):1894-1905. doi:10.1056/NEJMoa1915745

61. Finn RS, Qin S, Ikeda M, et al. IMbrave150: updated overall survival (OS) data from a global, randomized, open-label phase III study of atezolizumab (atezo) + bevacizumab (bev) versus sorafenib (sor) in patients (pts) with unresectable hepatocellular carcinoma (HCC). JCO. 2021;39(3_suppl):267. doi:10.1200/JCO.2021.39.3_suppl.267
62. Vogel A, Martinelli E, Vogel A, et al. Updated treatment recommendations for hepatocellular carcinoma (HCC) from the ESMO Clinical Practice Guidelines. Ann Oncol. 2021;32(6):801-805. doi:10.1016/j. annonc.2021.02.014

63. Patel SA, Minn AJ. Combination Cancer Therapy with Immune Checkpoint Blockade: mechanisms and Strategies. Immunity. 2018;48(3):417-433. doi:10.1016/j.immuni.2018.03.007

64. Li F, Zhao C, Wang L. Molecular-targeted agents combination therapy for cancer: developments and potentials: mTAs combination therapy for cancer. Int J Cancer. 2014;134(6):1257-1269. doi:10.1002/ijc.28261

65. Tada Y, Togashi Y, Kotani D, et al. Targeting VEGFR2 with Ramucirumab strongly impacts effector/ activated regulatory $\mathrm{T}$ cells and CD8+ T cells in the tumor microenvironment. $j$ Immunotherapy Cancer. 2018;6(1):106. doi:10.1186/s40425-018-0403-1

66. Chen DS, Hurwitz H. Combinations of Bevacizumab With Cancer Immunotherapy. Cancer J. 2018;24(4):193-204. doi:10.1097/ PPO.0000000000000327

67. Wallin JJ, Bendell JC, Funke R, et al. Atezolizumab in combination with bevacizumab enhances antigen-specific T-cell migration in metastatic renal cell carcinoma. Nat Commun. 2016;7(1):12624. doi:10.1038/ncomms 12624

68. FDA approves atezolizumab plus bevacizumab for unresectable hepatocellular carcinoma | FDA. Available from: https://www.fda. gov/drugs/resources-information-approved-drugs/fda-approves-atezo lizumab-plus-bevacizumab-unresectable-hepatocellular-carcinoma. Accessed October 4, 2021.

69. Herbst RS, Arkenau HT, Santana-Davila R, et al. Ramucirumab plus pembrolizumab in patients with previously treated advanced non-small-cell lung cancer, gastro-oesophageal cancer, or urothelial carcinomas (JVDF): a multicohort, non-randomised, open-label, phase 1a/b trial. Lancet Oncol. 2019;20(8):1109-1123. doi:10.1016/ S1470-2045(19)30458-9

70. Arkenau H, Martin-Liberal J, Calvo E, et al. Ramucirumab Plus Pembrolizumab in Patients with Previously Treated Advanced or Metastatic Biliary Tract Cancer: nonrandomized, Open-Label, Phase I Trial (JVDF). Oncol. 2018;23(12):1407. doi:10.1634/theoncologist.2018-0044

71. Bang YJ, Golan T, Dahan L, et al. Ramucirumab and durvalumab for previously treated, advanced non-small-cell lung cancer, gastric/gastro-oesophageal junction adenocarcinoma, or hepatocellular carcinoma: an open-label, phase $\mathrm{Ia} / \mathrm{b}$ study (JVDJ). Eur J Cancer. 2020;137:272-284. doi:10.1016/j.ejca.2020.06.007

72. Lin C, Yang T, Yen C, Cheng R, Liu J, Hsu C. Safety and Preliminary Efficacy of Ramucirumab in Combination with FoLfox4 in Patients with Advanced Hepatocellular Carcinoma: a Nonrandomized, OPENLABEL, Phase Ib Study. Oncol. 2020;25:12. doi:10.1002/onco.13550

73. Harding JJ, Zhu AX, Bauer TM, et al. A Phase Ib/II Study of Ramucirumab in Combination with Emibetuzumab in Patients with Advanced Cancer. Clin Cancer Res. 2019;25(17):5202-5211. doi:10.1158/1078-0432.CCR-18-4010

74. Harding JJ, Do RK, Yaqubie A, et al. Phase 1b study of galunisertib and ramucirumab in patients with advanced hepatocellular carcinoma. Cancer Med. 2021;10(9):3059-3067. doi:10.1002/cam4.3880

75. Imfinzi plus tremelimumab significantly improved overall survival in HIMALAYA Phase III trial in 1st-line unresectable liver cancer. Available from: https://www.astrazeneca.com/content/astraz/mediacentre/press-releases/2021/imfinzi-and-tremelimumab-improved-osin-liver-cancer.html. Accessed October 26, 2021. 


\section{Publish your work in this journal}

OncoTargets and Therapy is an international, peer-reviewed, open access journal focusing on the pathological basis of all cancers, potential targets for therapy and treatment protocols employed to improve the management of cancer patients. The journal also focuses on the impact of management programs and new therapeutic

Submit your manuscript here: https://www.dovepress.com/oncotargets-and-therapy-journal agents and protocols on patient perspectives such as quality of life, adherence and satisfaction. The manuscript management system is completely online and includes a very quick and fair peer-review system, which is all easy to use. Visit http://www.dovepress.com/ testimonials.php to read real quotes from published authors. 